\title{
Interprétation des profils de concentration pour des particules de sable en suspension de différentes tailles
}

\author{
Rafik ABSI ${ }^{1}$, Charlène LE GALL ${ }^{2}$, Stéphane MARCHANDON ${ }^{1}$, \\ Pierre-Antoine HAMMAD ${ }^{1}$ \\ ${ }^{1}$ Ecole de Biologie Industrielle, \\ 32, Bd du Port, 95094 Cergy Pontoise, France \\ r.absi@ebi-edu.com ; s.marchandon@ebi-edu.com;pa.hammad@ebi-edu.com \\ 2 Elcimaï Ingénierie, \\ 2 Bis Avenue Jean Jaurès, 77000 Melun, France \\ clegall@elcimai.com
}

\begin{abstract}
Résumé :
La forme des profils verticaux de concentration des sédiments en suspension sur un fond de rides, qui peut être concave ou convexe, dépend de la taille des sédiments. Pour améliorer la prédiction de ces profils, nous considérons d'une part, le modèle de longueur de mélange finie avec différentes descriptions du mélange turbulent et d'autre part, l'équation d'advection-diffusion classique basée sur l'approximation de Fick avec une description appropriée du paramètre $\beta$ (rapport entre diffusivité et viscosité turbulente). L'analyse détaillée des données de mesures a permis d'identifier deux couches distinctes, une première proche du fond où le profil de concentration est concave et une seconde plus éloignée où le profil est convexe. La limite de passage d'une couche à l'autre dépend de la taille des sédiments.
\end{abstract}

\section{Mots-clés:}

SEDIMENTS - MELANGE TURBULENT - PROFILS DE SEDIMENTS TAILLES DES PARTICULES

\begin{abstract}
:
The shape of suspended sediment concentration vertical profiles over a wave ripples, which is upward concave or convex, depends on sand grain sizes. In order to improve the prediction of these profiles, we consider on the hand the finite mixing length model with different descriptions of turbulent mixing and on the other hand the classical advection-diffusion equation based on Fick's approximation with an appropriate description of the parameter $\beta$ (ratio between sediment diffusivity and eddy viscosity). A detailed analysis of experimental data shows two layers, a first near the bottom where the concentration profile is
\end{abstract}


upward concave and a second where the profile is upward convex. The limit between these two layers depends on sediments size.

\section{Mots-clés:}

SEDIMENT - TURBULENT MIXING -SEDIMENT PROFILES - PARTICLE SIZE

\section{Introduction}

Le transport sédimentaire côtier, résultant de l'hydrodynamique (houles, marées, courants), est un phénomène critique qui provoque l'évolution de la ligne de côte et des fonds marins. La compréhension de ce phénomène a un intérêt environnemental et économique fort.

Les modèles actuellement utilisés pour le calcul de la concentration des sédiments en suspension sur un fond de rides présentent quelques lacunes. En effet, ils ne permettent pas de prédire les profils de concentration concave et convexe qu'on rencontre pour des particules de sables de différentes tailles. Ces profils ont été observés in situ et en laboratoire (McFETRIDGE \& NIELSEN, 1985) sur un fond constitué de rides. Cette faiblesse au niveau de ces modèles est étroitement liée au problème fondamental de l'interaction entre les particules de sable et l'écoulement turbulent.

L'objectif de notre communication est de contribuer à améliorer nos capacités de calcul des profils de concentration des sédiments en suspension. Pour cela, nous utiliserons une modélisation analytique du mélange turbulent avec d'une part l'équation d'advection-diffusion classique basée sur l'approximation de Fick avec une nouvelle fonction pour le paramètre $\beta$ (rapport entre diffusivité et viscosité turbulente) et d'autre part le modèle de longueur de mélange finie (NIELSEN \& TEAKLE, 2004).

\section{$2 \quad$ Modélisation des profils de concentration}

En écoulement oscillant (cas d'une houle), les sédiments en suspension sont soumis à un flux de sédimentation, descendant, et à un flux net ascendant dû au mélange turbulent. Le flux $\vec{q}_{s}$ peut s'écrire en fonction de la concentration: $\vec{q}_{s}=c(z) \cdot \vec{w}_{\mathrm{s}}$; où $w_{s}$ est la vitesse de chute des sédiments et $z$ est la distance par rapport au fond. Pour établir le profil moyen de concentration $c(z)$ des sédiments à l'équilibre, il suffit d'écrire que le flux de sédimentation $\vec{q}_{s}$ compense exactement le flux de mélange $\vec{q}_{m}$. L'équilibre des flux impose la relation $\vec{q}_{s}+\vec{q}_{m}=\overrightarrow{0}$, d'où : $q_{m}-w_{s} c(z)=0$

\subsection{Modèle classique basé sur l'approximation de Fick}

L'écriture du flux de mélange $\vec{q}_{m}$ peut être simplement obtenue en écrivant une proportionnalité entre $\vec{q}_{m}$ et le gradient de concentration, ou loi de Fick, de même 
forme que celle obtenue dans le cas de la diffusion moléculaire :

$q_{m}=-D_{t} \frac{d c}{d z}$

Où $D_{t}$ est la diffusivité turbulente. Soit, à partir des écritures (1) et (2), nous obtenons l'équation d'advection-diffusion classique :

$$
\frac{d c}{d z}=-\frac{w_{s}}{D_{t}} c
$$

\subsection{Modèle de longueur de mélange finie}

Dans le modèle de longueur de mélange finie proposé par NIELSEN \& TEAKLE (2004), le flux de mélange ascendant s'écrit

$q_{m}=w_{m} \cdot\left[c\left(z-l_{m} / 2\right)-c\left(z+l_{m} / 2\right)\right]$

où : $w_{m}$ est la vitesse de mélange et $l_{m}$ la longueur de mélange. Le développement en série de Taylor donne :

$q_{m}=-w_{m} \cdot l_{m}\left[\frac{d c}{d z}+\frac{l_{m}{ }^{2} \cdot d^{3} c}{24 d z^{3}}+\ldots\right]$

Le remplacement dans l'équation (1), donne :

$-w_{m} \cdot l_{m}\left[\frac{d c}{d z}+\frac{l_{m}{ }^{2} \cdot d^{3} c}{24 d z^{3}}+\ldots\right]-w_{s} c(z)=0$

NIELSEN \& TEAKLE (2004) ont écrit l'équation (6), en se limitant aux deux premiers termes du développement, sous la forme d'une équation différentielle ordinaire du troisième ordre :

$\frac{l_{m}^{2} \cdot d^{3} c}{24 d z^{3}}+\frac{d c}{d z}+\frac{w_{s}}{w_{m} \cdot l_{m}} c(z)=0$

Nous pouvons remarquer que nous obtenons l'équation (3) à partir de l'équation (7), en négligeant le premier terme de (7) et en décrivant le mélange turbulent non par le produit $w_{m} \cdot l_{m}$, mais par $D_{t}$.

\section{$3 \quad$ Le mélange turbulent}

La résolution des équations différentielles (3) et (7) nécessite la connaissance du mélange turbulent, qui passe soit par la diffusivité turbulente $D_{t}$ (pour l'éq. 3), soit par le produit $w_{m} \cdot l_{m}$ (pour l'éq. 7). Ce produit représente la viscosité turbulente $v_{t}$, qui est reliée à la diffusivité turbulente par le paramètre $\beta$ : $D_{t}=\beta . v_{t}$. Nous écrivons la viscosité turbulente comme :

$v_{t}=w_{m} \cdot l_{m}=C_{\mu}^{1 / 4} \cdot \sqrt{k} \cdot l_{m}$

où $k$ est l'énergie cinétique de la turbulence et $C_{\mu}^{1 / 4}=0,09$ (coefficient du modèle $k-\varepsilon)$. Le profil de vitesse de mélange $w_{m}=C_{\mu}^{1 / 4} \cdot \sqrt{k}$ est donné par la solution analytique générale de l'équation de l'énergie cinétique de la turbulence obtenue 
dans ABSI (2008a). La solution particulière, qui correspond à l'équilibre entre production et dissipation de l'énergie cinétique turbulente, est similaire d'un coté à la fonction semi-empirique de NEZU \& NAKAGAWA (1993) et de l'autre à la relation empirique utilisée par NIELSEN \& TEAKLE (2004) qui s'appuie sur les mesures de DU TOIT \& SLEATH (1981) sur un fond de rides.

$$
\frac{k}{U_{f}^{2}}=D_{2} \cdot \exp \left(-2 . C_{1} \xi\right)=\frac{1}{C_{\mu}^{1 / 2}} \cdot \alpha^{2} \cdot \exp \left(-2 . C_{1} \xi\right)
$$

où, $\xi=z / h$, avec $h$ pouvant représenter soit la hauteur d'eau soit l'épaisseur de la couche limite. $D_{2}, C_{1}$ et $\alpha$ sont des coefficients, et $U_{f}$ est la vitesse de frottement. La vitesse de mélange s'écrit donc :

$w_{m}=\alpha \cdot U_{f} \cdot \exp \left(-C_{1} \xi\right)=w_{m}\left(z_{l}\right) \cdot \exp \left(-\frac{z}{L_{w}}\right)$

où : $w_{m}\left(z_{l}\right)=\alpha U_{f} ; z_{l}$ un niveau à partir duquel la vitesse de mélange (et donc $k$ ) commence à décroitre (ABSI, 2008a), et $L_{w}=h / C_{1}$. NIELSEN \& TEAKLE (2004) ont pris la vitesse de mélange donnée par l'éq. (10) et une longueur de mélange qui croît linéairement $l_{m 1}(z)=\lambda \cdot z$. Leur viscosité turbulente s'écrit alors

$v_{t}=l_{m} \cdot w_{m}=\lambda z \cdot w_{m}\left(z_{l}\right) \cdot \exp \left(-\frac{z}{L_{w}}\right)$

Le choix de NIELSEN \& TEAKLE pour $w_{m}$ et $l_{m}$ donne un profil de vitesse non logarithmique. Si on suppose un profil de vitesse logarithmique, la longueur de mélange s'écrit selon ABSI (2008b)

$l_{m 2}(z)=\alpha \cdot \kappa \cdot z \cdot e^{\left(-\frac{z}{L_{w}}\right)}$

Les équations (10) et (12) permettent d'écrire la viscosité turbulente comme suit :

$v_{t}=\alpha^{2} \cdot \kappa \cdot U_{f} \cdot z \cdot e^{\left(-2 \frac{z}{L_{w}}\right)}=\alpha^{2} \cdot \kappa \cdot U_{f} \cdot z \cdot e^{\left(-2 C_{1} \frac{z}{h}\right)}$

$4 \quad$ Calcul des profils de concentration pour les sédiments en suspension

NIELSEN \& TEAKLE (2004) ont comparé les solutions obtenues à partir des équations (7) et (3) pour le cas d'un écoulement oscillant (houle) sur un fond de rides pour deux types de particules de sable, des petites particules $w_{s}=0.65 \mathrm{~cm} / \mathrm{s}$ et des plus grosses $w_{s}=6.1 \mathrm{~cm} / \mathrm{s}$. La figure (1) présente les profils de concentration moyenne, les données de mesures sont celles de McFETRIDGE \& NIELSEN (1985). Les traits pleins représentent les solutions de l'équation (7) avec $l_{m}=\lambda z$, $\lambda=1, z_{l}=0.005 \mathrm{~m}, w_{m}\left(z_{l}\right)=0.025 \mathrm{~m} / \mathrm{s}, L_{w}=0.022 \mathrm{~m}$, tandis que les traits en tiretspointillés représentent les solutions de l'équation (3). 


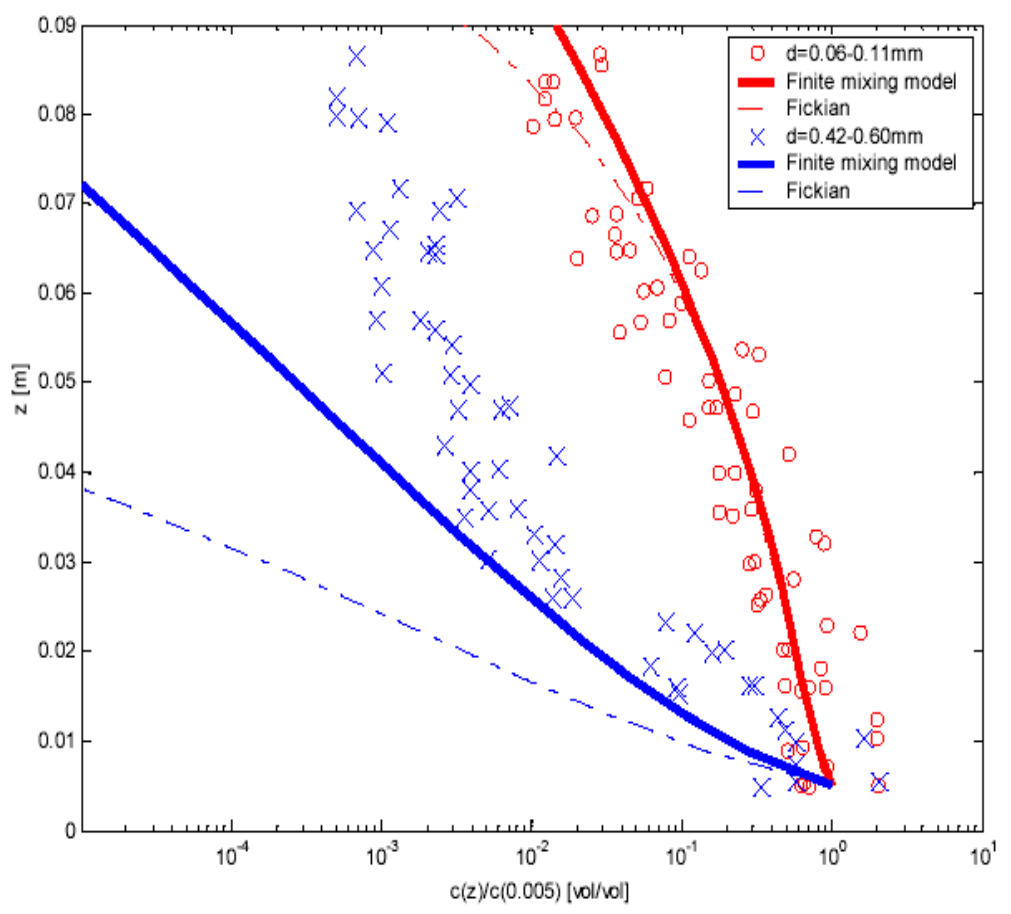

Figure 1. Profil de concentration moyenne sur un fond de rides pour des petites particules $(o) w_{s}=0.65 \mathrm{~cm} / \mathrm{s}$ et des grosses particules $(\times) w_{s}=6.1 \mathrm{~cm} / \mathrm{s}($ symboles mesures). Traits pleins: solution de (7) avec $l_{m}=\lambda z \quad(\lambda=1)$ et $z_{l}=0.005 m$, $w_{m}\left(z_{l}\right)=0.025 \mathrm{~m} / \mathrm{s}, \quad L_{w}=0.022 \mathrm{~m}$. tirets-pointillés, solution de l'éq. (3) (Figure d'après NIELSEN \& TEAKLE, 2004).

\subsection{Profils obtenus avec le modèle de longueur de mélange finie}

Comme précisé dans (ABSI, 2005), certaines équations de longueur de mélange permettent d'améliorer les prédictions du modèle de longueur de mélange finie. La solution (9) pour l'énergie cinétique de la turbulence permet de développer des équations pour la longueur de mélange. Nous avons proposé d'autres équations pour la longueur de mélange. Une première, noté $l_{m 3}$, qui s'écrit selon ABSI (2005)

$l_{m 3}(z)=\kappa \cdot z_{0} \cdot e^{\left(3 \frac{z-z_{0}}{L_{w}}\right)}$

et une autre obtenue à partir d'une extension de l'hypothèse de similitude de Von Karman qui s'écrit selon ABSI (2006)

$$
l_{m 4}(z)=\kappa \cdot\left[L_{w}-\left(L_{w}-z_{0}\right) \cdot e^{\left(-\frac{\left(z-z_{0}\right)}{L_{w}}\right)}\right]
$$

Le profil vertical de $l_{m 3}$ est convexe. La figure 2 représente le profil de concentration obtenu après résolution de l'équation (7) avec le profil convexe $l_{m 3}$. Cette figure montre que le profil de concentration $c(z)$ obtenu est concave pour les 
Dynamique sédimentaire et transport des particules

grosses particules. Le profil vertical de $l_{m 4}$ est concave.

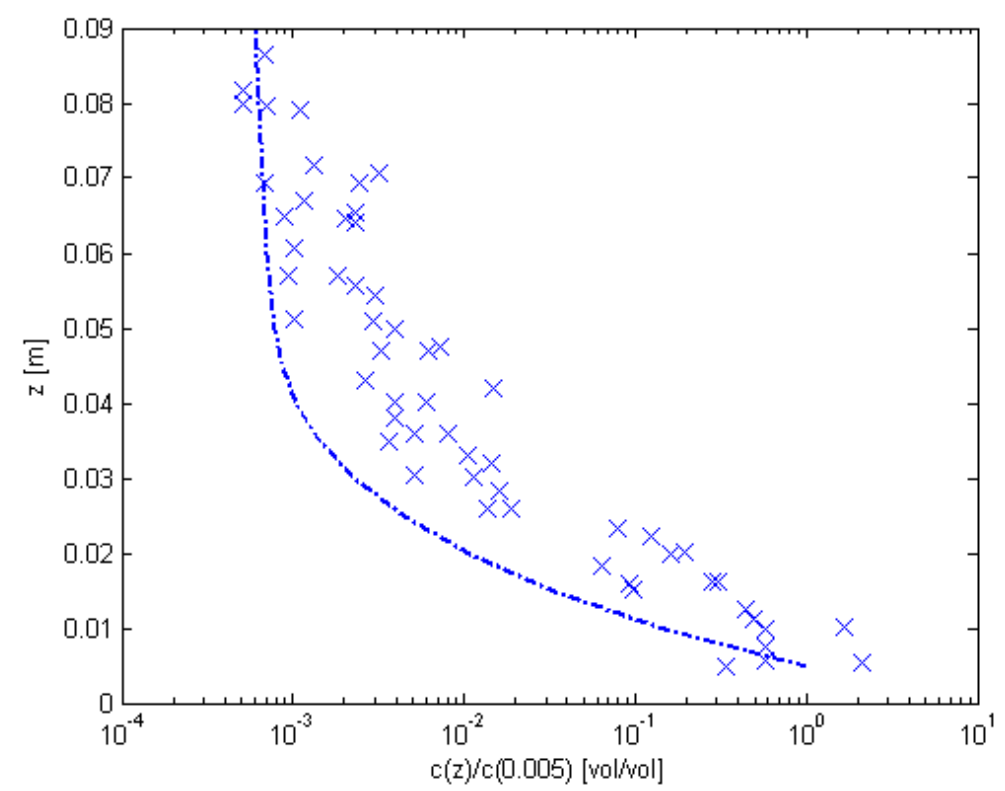

Figure 2. Profil de concentration moyenne sur un fond de rides pour des particules grosses $\left(w_{s}=6.1 \mathrm{~cm} / \mathrm{s}\right)$. Courbe : solution de l'éq. (7) avec l'éq. (14) ; $(\lambda=1) ; L_{w}=0.05 \mathrm{~m}, z_{l}=0.005 \mathrm{~m} ; w_{m}\left(z_{l}\right)=0.025 \mathrm{~m} / \mathrm{s} . \times$ : mesures.

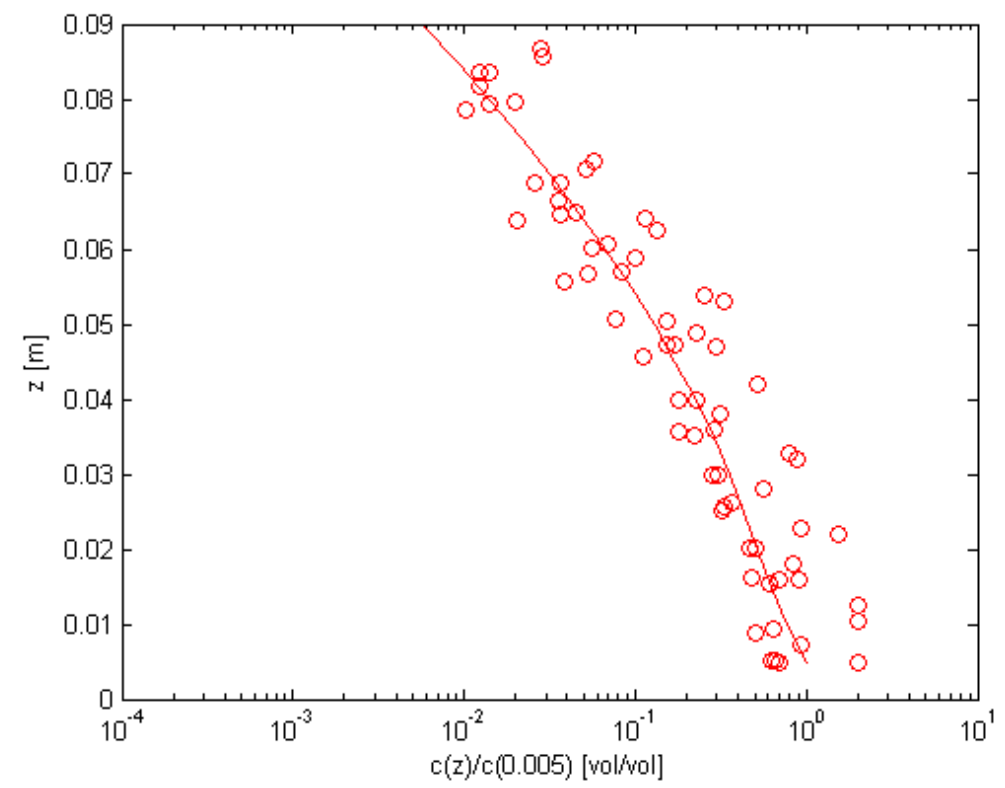

Figure 3. Profil de concentration moyenne sur un fond de rides pour des particules fines $\left(w_{s}=0.65 \mathrm{~cm} / \mathrm{s}\right)$. courbe : solution de l'éq. (7) avec l'éq. (15) ; $(\lambda=1) ; L_{w}=0.022 \mathrm{~m}, z_{l}=0.005 \mathrm{~m} ; w_{m}\left(z_{l}\right)=0.025 \mathrm{~m} / \mathrm{s}$. o: mesures. 
La figure 3 représente le profil de concentration obtenu après résolution de l'équation (7) avec le profil concave $l_{m 4}$; cette figure montre que le profil de concentration $c(z)$ est convexe pour les particules fines. Il apparaît donc essentiel de distinguer les particules fines des grosses, nous avons considéré donc deux descriptions différentes du mélange turbulent.

En effet, le profil de longueur de mélange convexe est capable de prédire un profil de concentration concave et inversement le profil de longueur de mélange concave est capable de prédire un profil de concentration convexe. Dans cette partie, nous avons supposé que la taille des sédiments affecte le mélange turbulent. Le mélange des particules qui passe par le produit $w_{m} \cdot l_{m}$ semble différent (le produit $w_{m} \cdot l_{m}$ pour les petites est différent du produit pour les grosses).

\subsection{Profils obtenus avec le modèle classique}

Il est possible aussi d'avoir une bonne prédiction des profils de concentration aussi bien pour les petites particules que pour les grosses à partir de l'équation (3), avec la même description pour $w_{m}$ et $l_{m}$ commune aux petites et aux grosses particules. La viscosité turbulente est la même quelle que soit la taille des particules, cependant la diffusivité turbulente $D_{t}$, qui est donnée par $D_{t}=\beta . v_{t}$, est différente. L'effet de la taille des particules sera pris en compte par le paramètre $\beta$.

Nous avons proposé (ABSI, 2008b) pour le paramètre $\beta$ une fonction empirique, à partir des expériences de GRAF et CELLINO (2002), dépendant de la distance $z$

$\beta(z)=\beta_{0} \cdot f_{\beta}(z) \quad$ avec : $f_{\beta}(z)=e^{C_{\beta} \cdot \xi}$

Avec l'équation (13) pour $v_{t}$, la diffusivité turbulente s'écrit donc :

$D_{t}=\beta \cdot v_{t}=\beta_{0} e^{C_{\beta} \cdot \xi} \cdot \alpha^{2} \kappa U_{f} z e^{-2 C_{1} \frac{z}{h}}=\beta_{0} \alpha^{2} \kappa U_{f} z e^{-\left(2 C_{1}-C_{\beta}\right) \frac{z}{h}}$

qu'on peut écrire sous la forme suivante :

$D_{t}=\alpha_{D t} z e^{-\frac{z}{h_{D t}}}$

avec : $\alpha_{D t}=\beta_{0} \alpha^{2} \kappa U_{f}$ et $h_{D t}=\frac{h}{\left(2 C_{1}-C_{\beta}\right)}$.

Les résultats de l'équation (3) avec $D_{t}$ donné par l'équation (18) sont présentés dans la figure 4. 


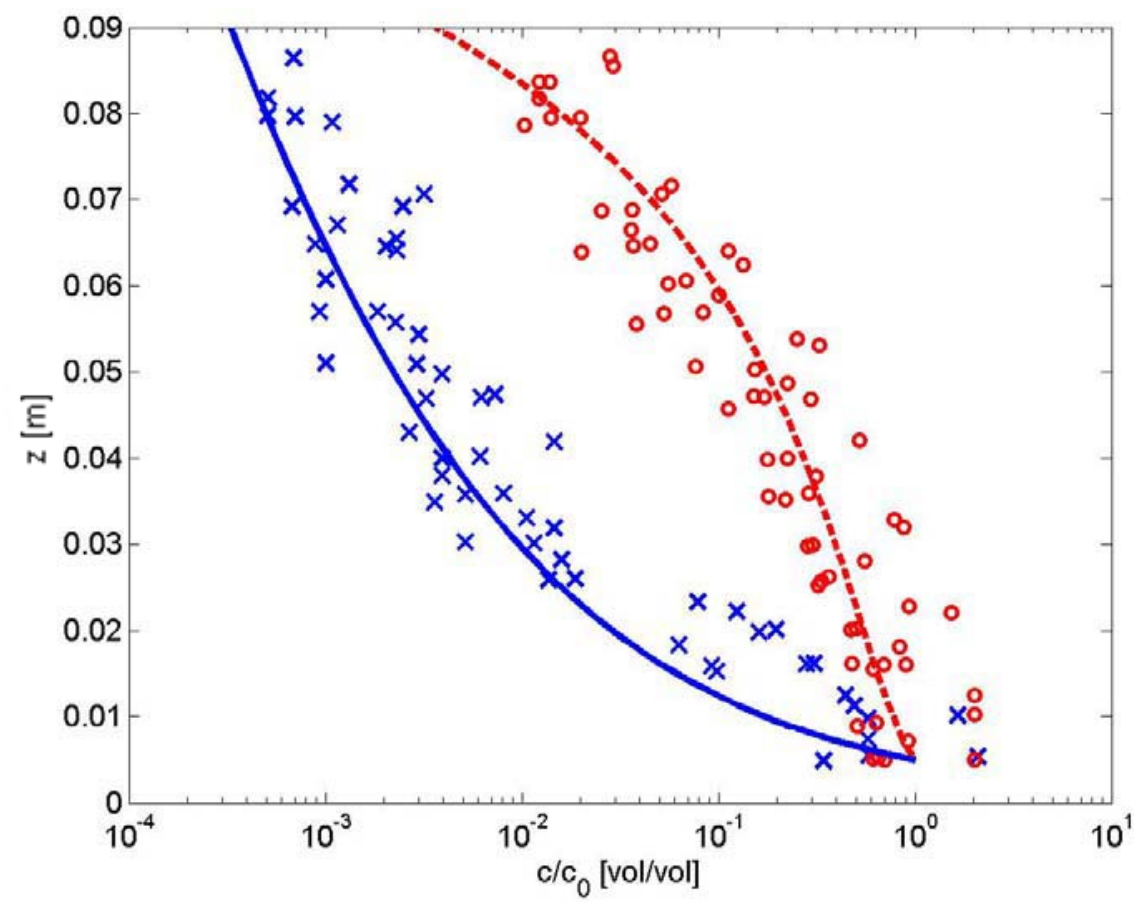

Figure 4. Profil de concentration moyenne sur un fond de rides pour des petites particules (o) $w_{s}=0.65 \mathrm{~cm} / \mathrm{s}$ et des grosses ( $\times$ ) $w_{s}=6.1 \mathrm{~cm} / \mathrm{s}$ (mesures (McFETRIDGE \& NIELSEN, 1985)). Courbes: solutions de (3) avec $\alpha_{D t}=0.025 \mathrm{~m} / \mathrm{s}$. Trait plein : $h_{D t}=0.24 \mathrm{~m}$. Tirets : $h_{D t}=0.022 \mathrm{~m}$.

\subsection{Interprétation et Discussions}

La concavité/convexité du profil de concentration est liée à $\frac{d^{2} \ln c}{d z^{2}}$, tandis que la croissance/décroissance de la diffusivité turbulente est liée à $\frac{d D_{t}}{d z}$. Pour comprendre le lien entre concavité/convexité du profil de concentration et croissance/décroissance de la diffusivité turbulente, nous avons besoin d'une relation mathématique entre $\frac{d^{2} \ln c}{d z^{2}}$ et $\frac{d D_{t}}{d z}$. Nous avons : $\frac{d \ln c}{d z}=\frac{1}{c}\left(\frac{d c}{d z}\right)$ et avec l'équation (1): $\frac{d \ln c}{d z}=-\frac{w_{s}}{D_{t}}$. La dérivée seconde de $\ln c$ s'écrit alors (ABSI 2008b) :

$\frac{d^{2} \ln c}{d z^{2}}=\frac{w_{s}}{D_{t}^{2}} \frac{d D_{t}}{d z}$

Puisque le terme $\frac{w_{s}}{D_{t}^{2}}$ est toujours positif, l'équation (19) montre que $\frac{d^{2} \ln c}{d z^{2}}$ et 
$\frac{d D_{t}}{d z}$ ont le même signe. Par conséquent une diffusivité turbulente croissante entraîne un profil de concentration concave tandis qu'une diffusivité turbulente décroissante entraine un profil de concentration convexe.

Notre analyse fine des mesures de McFETRIDGE \& NIELSEN 1985 (voir figure 5) montre que pour les grosses particules il y a deux zones, une près du fond où le profil $c(z)$ est convexe et une autre plus éloignée où $c(z)$ est concave. Pour les particules fines, nous n'observons qu'un seul profil convexe. L'analyse d'autres données expérimentales sur un fond plat confirme qu'il y a deux couches, une première proche du fond où le profil $c(z)$ est convexe et une seconde où $c(z)$ est concave plus éloignée. Nous avons remarqué également que le seuil entre les deux types de profils, la limite de passage (à partir du fond) d'une couche à un autre, est plus importante pour les fines particules que pour les grosses (ABSI, 2006).

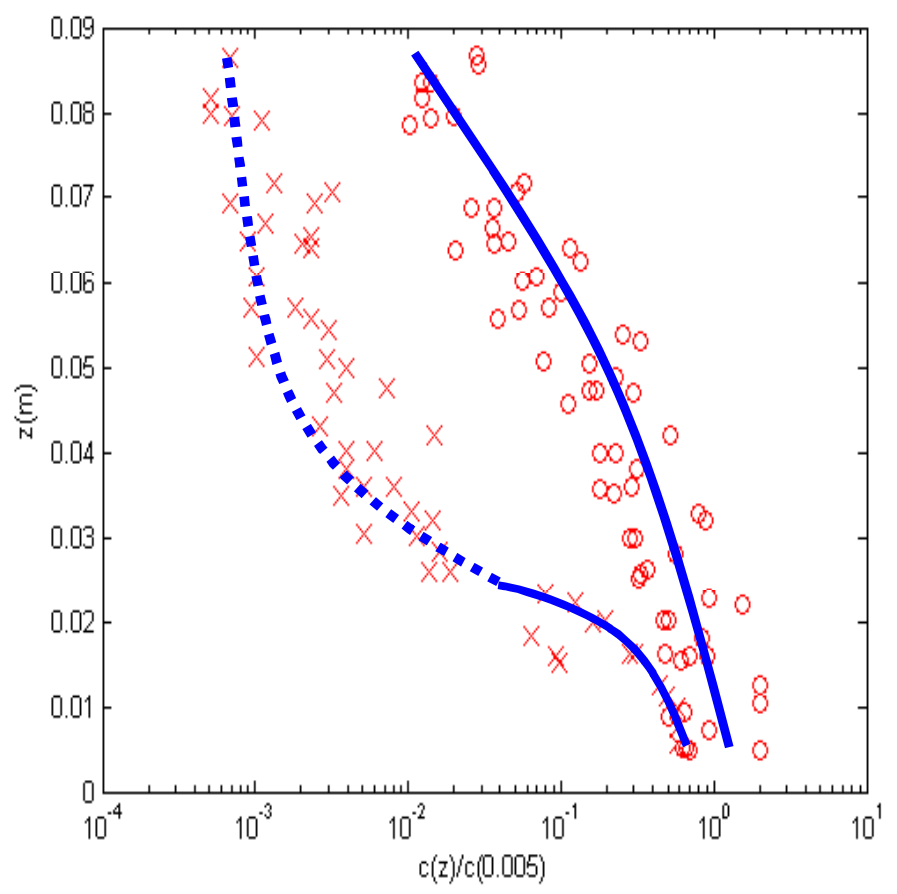

Figure 5. Profil de concentration moyenne sur un fond de rides. o, sable fin $w_{s}=0.65 \mathrm{~cm} / \mathrm{s} ; \times$, sable plus gros $w_{s}=6.1 \mathrm{~cm} / \mathrm{s}$.

Comme la limite entre les deux couches dépend de la taille des particules de sable, nous avons suggéré dans un premier temps que le mélange turbulent dépend aussi de la taille des particules de sable. Cependant, ceci ne semble pas être la seule explication. En effet, proche du fond, les profils sont liés aussi au dépôt, le phénomène de sédimentation prédomine. Le profil concave serait donc lié au mélange turbulent, alors que le profil convexe serait plus lié au phénomène de sédimentation qui est plus important près du fond. C'est pourquoi la taille des 
particules impacte fortement les paramètres des profils et notamment la hauteur qui définit le seuil entre les deux types de profils : concave et convexe.

\section{Conclusion}

Pour améliorer la prédiction des profils de concentration des sédiments en suspension sur un fond de rides pour des sédiments de différentes tailles, nous avons considéré d'une part le modèle de longueur de mélange finie et de l'autre l'équation d'advection-diffusion classique basée sur l'approximation de Fick.

Le modèle de longueur de mélange finie a nécessité deux descriptions différentes pour le mélange turbulent, tandis que l'équation d'advection-diffusion classique a nécessité une fonction adéquate pour le paramètre $\beta$. La forme des profils de concentrations a été interprétée par une relation mathématique entre $\frac{d^{2} \ln c}{d z^{2}}$ et $\frac{d D_{t}}{d z}$. Une analyse fine a permis d'identifier deux couches distinctes, une première proche du fond où le profil de concentration est concave et une seconde plus éloignée où le profil est convexe. La hauteur par rapport au fond de la limite de passage d'une couche à l'autre dépend de la taille des particules de sable.

\section{$6 \quad$ Références bibliographiques}

1 ABSI, R. (2005). Comment on Turbulent diffusion of momentum and suspended particles: A finite-mixing-length-theory. Physics of Fluids, 17(7), pp. 079101,1-2.

2 ABSI, R. (2006). On the effect of sand grain size on turbulent mixing, Proceedings of $30^{\text {th }}$ Int. Conf. on Coastal Engineering, ASCE, San Diego, Jane McKee Smith (editor), World Scientific, pp. 3019-3029.

3 ABSI, R. (2008a). Analytical solutions for the modeled k-equation, Journal of Applied Mechanics, Transactions of ASME, 75(4), pp. 044501,1-4.

4 ABSI, R. (2008b). Vertical dispersion of fine and coarse sediments over wave ripples, Rapport interne EBI-0508.

5 DU TOIT, C. G. and SLEATH, J.F.A. (1981). Velocity measurements close to rippled beds in oscillatory flow. J. Fluid Mech., 112, pp. 71-96.

6. GRAF, W. H., and CELLINO, M., 2002. Suspension flows in open channels: experimental study. Journal of Hydraulic Research, 40(4), pp. 435-447.

7 McFETRIDGE, W. F. and NIELSEN, P. (1985). Sediment suspension by non-breaking waves. Technical Report No. Tech Rep COEL, 85/005, Dept Coastal \& Oceanographical Eng, Univ. of Florida, $131 \mathrm{p}$.

8 NEZU, I., and NAKAGAWA, H. (1993). Turbulence in open-channel flow, A.A. Balkema, ed., Rotterdam, The Netherlands.

9 NIELSEN, P., and TEAKLE, I.A.L. (2004). Turbulent diffusion of momentum and suspended particles: A finite-mixing-length-theory. Physics of Fluids, 16(7), pp. 2342-2348. 